EPJ Web of Conferences 66, 01001 (2014)

DOI: $10.1051 /$ epjconf/ 20146601001

(C) Owned by the authors, published by EDP Sciences, 2014

\title{
Structure and spin of the nucleon
}

\author{
H. Avakian ${ }^{1, a}$ \\ ${ }^{1}$ Thomas Jefferson National Accelerator Facility, 12000 Jefferson Ave. Suite 5,Newport News, VA 23606
}

\begin{abstract}
.
Parton distribution functions, describing longitudinal momentum, helicity and transversity distributions of quarks and gluons, have been recently generalized to account also for transverse degrees of freedom. Two new sets of more general distributions, Transverse Momentum Distributions and Generalized Parton Distributions, were introduced to describe transverse momentum and space distributions of partons.

Great progress has been made since then in measurements of different Single Spin Asymmetries (SSAs) in semi-inclusive and hard exclusive processes providing access to TMDs and GPDs, respectively. Facilities world-wide involved in studies of the 3D structure of nucleon include HERMES, COMPASS, BELLE, BaBar, Halls A, B, and C at JLab, and PHENIX and STAR at RHIC (BNL). TMD studies in the Drell-Yan process are also becoming an important part of the program of hadron scattering experiments. Studies of TMDs are also among the main driving forces of the JLab $12-\mathrm{GeV}$ upgrade project, several of the forward upgrade proposals of STAR and PHENIX at RHIC, and future facilities, such as the Electron Ion Collider (EIC), FAIR in Germany, and NICA in Russia. In this contribution we present an overview of the latest developments in studies of parton distributions and discuss newly released results, ongoing activities, as well as some future measurements.
\end{abstract}

\section{Introduction}

Since the late 60s many theoretical and experimental groups dedicated their time to study the quarkgluon structure of nucleons and nuclei in terms of collinear ( "integrated") parton distributions (PDFs) of hadrons. One of the most surprising results is the unexpectedly small fraction of the proton's spin that is due to the contribution from quarks and antiquarks directing attention toward orbital motion of partons. Transverse space distributions of partons, encoded in Generalized Parton Distributions (GPDs), and transverse momentum dependent distributions, encoded in Transverse Momentum Distributions (TMDs), have been widely recognized as key objectives of the JLab 12-GeV upgrade [1] and the polarized pp program at RHIC [2] as well as a driving force behind the construction of the EIC [3]. The information on QCD-dynamics inside hadrons, as encoded in GPDs and TMDs, is much richer than what one can learn from collinear PDFs. These 3D PDFs provide the information on the orbital structure, which is not accessible through regular PDFs and are expected to play a crucial role in explaining the spin structure of the nucleon. TMD and GPD distributions (see Table 1) describe partons with certain polarizations in nucleons in independence of the polarization state.

\footnotetext{
a e-mail: avakian@jlab.org
} 


\begin{tabular}{|c|c|c|c|}
\hline N/q & $\mathrm{U}$ & $\mathrm{L}$ & $\mathrm{T}$ \\
\hline $\mathrm{U}$ & $\mathbf{f}_{1}$ & & $h_{1}^{\perp}$ \\
\hline $\mathrm{L}$ & & $\mathrm{g}_{1 \mathrm{~L}}$ & $h_{1 L}^{\perp}$ \\
\hline $\mathrm{T}$ & $f_{1 T}^{\perp}$ & $g_{1 T}$ & $h_{1}, h_{1 T}^{\perp}$ \\
\hline
\end{tabular}

\begin{tabular}{|c|c|c|c|}
\hline & $U$ & $L$ & $T$ \\
\hline$U$ & $\mathcal{H}$ & & $\mathcal{E}_{T}$ \\
\hline$L$ & & $\widetilde{\mathcal{H}}$ & $\widetilde{\mathcal{E}}_{T}$ \\
\hline$T$ & $\mathcal{E}$ & $\widetilde{\mathcal{E}}$ & $\mathcal{H}_{T}, \widetilde{\mathcal{H}}_{T}$ \\
\hline
\end{tabular}

Table 1. Leading twist TMD distribution functions (left) and GPDs (right). The U,L,T correspond to unpolarized, longitudinally polarized, and transversely polarized nucleons (rows) and quarks (columns)

One of the most prominent applications is the Ji's sum rule [4], providing access to the elusive orbital angular momentum. The integral is weighted by $x$ and calculated at any longitudinal momentum transfer $\xi$ and vanishing momentum transfer $t$.

$$
J=\lim _{t \rightarrow 0} \sum_{q=u, d, s} \frac{1}{2} \int_{-1}^{1} d x x\left\{H^{q}(x, \xi, t)+E^{q}(x, \xi, t)\right\}
$$

Recently there was a great progress in the proper definition of quark and gluon contributions to the proton spin. The gauge invariant interpretation of $\Delta g$ as the gluon spin contribution has been identified. It has been shown, however, that to access the canonical orbital angular momentum one has to extract experimentally either the phase space Wigner distribution [6] or particular twist-3 distributions [5]. The difference between different decompositions can be interpreted as the change in the quark orbital angular momentum due to final state interactions as the quark leaves the target in a DIS experiment [7].

Wide kinematic coverage of large acceptance detectors allows studies of exclusive (GPDs) and semi-inclusive (TMDs) processes providing complementary information on the transverse structure of nucleon. One of the cleanest processes to access GPDs is Deeply Virtual Compton Scattering (DVCS), in which one quark of the nucleon absorbs a virtual photon producing a real photon with the nucleon left intact. DVCS is most suitable for studying GPDs at moderate energies and in the valence quark regime. At low beam energies, the cross section for DVCS is small and masked by the more copious production of photons from the Bethe-Heitler $(\mathrm{BH})$ process. However, DVCS observables are only sensitive to chiral-even GPDs describing unpolarized and longitudinally polarized quarks. Hard exclusive production of light mesons allows to filter different GPDs by different final state hadrons $[8,9]$. In addition they provide a unique possibility to access "transversity" or chiralodd GPDs through measurements of different azimuthal moments of the cross section. In the handbag approach developed recently by Liuti and Goldstein [10] and Kroll and Goloskokov [11, 12] the helicity amplitudes depend on hard partonic subprocess and the GPD and the beam spin asymmetries of exclusive pions combined with other spin and azimuthal asymmetries, which can provide a unique possibility to access the elusive transversity GPDs.

\section{Collinear parton distributions}

Due to the dominance of gluon induced hard scattering processes at RHIC, longitudinally polarized proton-proton collisions studied by PHENIX and STAR are currently the best source of information on the gluon polarization, $\Delta g$. Measurements of the double helicity asymmetry, $A_{L L}$, of neutral pions and jets, at PHENIX and STAR respectively, indicate a sizable positive $\Delta g$ (see Figure 1) in the accessible range of $x(0.05<x<0.2)$. The uncertainties for $\Delta g(x)$, however, remain significant in the presently unmeasured small $x$ region, preventing a reliable determination of the full integral. 
Most of the measurements of the polarized light sea-quark distribution have been performed in polarized semi-inclusive DIS (SIDIS) by the SMC, HERMES, and COMPASS collaborations by identifying hadrons in the final state. The analysis of SIDIS data relies on quantitative understanding of the fragmentation of quarks and antiquarks into observable final-state hadrons and the uncertainties in the polarized antiquark PDFs separated by flavor so far remain relatively large.

Significant parity-violating single-spin asymmetries $\left(A_{L}\right)$ in longitudinally polarized $p p$ collisions have been observed recently at RHIC. The coupling of the $W s$ to the weak charge provides direct access to quark flavor allowing studies of the spin-flavor structure of sea quarks inside the proton. The STAR preliminary results on $A_{L}^{W^{ \pm}}$taken during 2012 shown in Figure 1 (right) have been already included in the pQCD-fit and improved significantly the determination of the polarization of the light sea quarks (DSSV++).
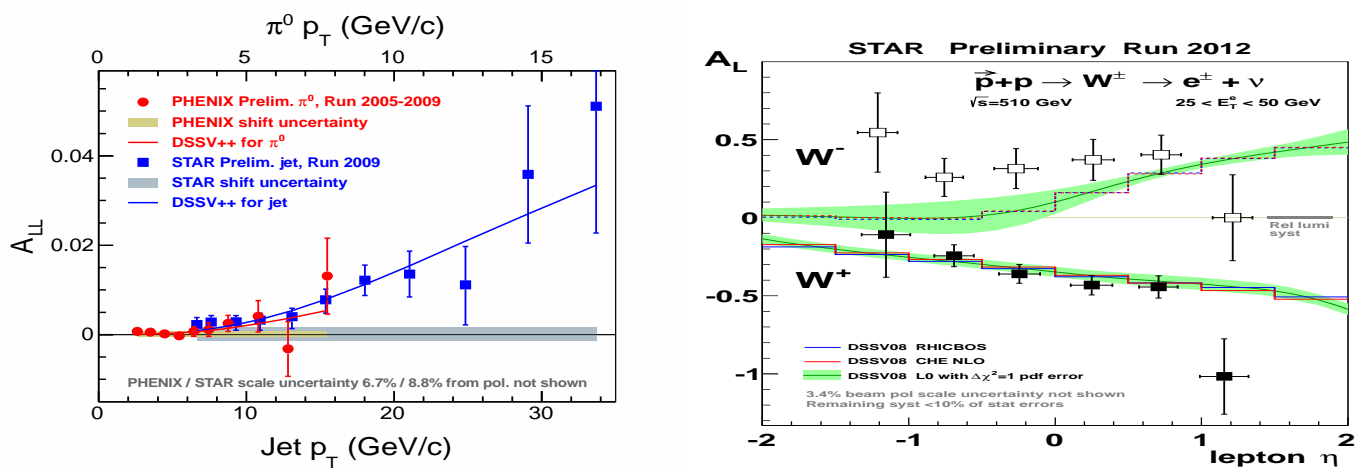

Figure 1. Preliminary 2009 data compared to the DSSV++ fit (left) and the single spin asimmetry, $A_{L}$, for $W^{ \pm}$as measured by STAR in 2012 (right). The $\eta$ is the charged lepton pseudorapidity in the CMS frame $(\eta=$ $\ln \left[\tan \left(\theta_{l} / 2\right)\right]$, where $\theta_{l}$ is the polar angle).

\section{Spin-azimuthal asymmetries}

In recent years, measurements of azimuthal moments of polarized hadronic cross sections in hard processes have emerged as a powerful tool to probe nucleon structure. Several experimental groups are currently trying to pin down various effects related to the nucleon structure through studies of spinazimuthal asymmetries in SIDIS (HERMES at DESY [13-16], COMPASS at CERN [17], Jefferson Lab [18-20]) polarized proton-proton collisions (PHENIX, STAR and BRAHMS at RHIC) [21, 22], and electron-positron annihilation (Belle at KEK and BaBar at SLAC) [23]. Azimuthal distributions of final state particles in SIDIS, in particular, are sensitive to the orbital motion of quarks and play an important role in the study of transverse momentum distributions (TMDs) of quarks in the nucleon.

Significant azimuthal moments in leptoproduction $\left(A_{U U}^{\cos \phi}\right)$, which have been measured in SIDIS already by EMC collaboration [24], were reproduced by latest measurements at CERN, HERMES and JLab $[19,20,25,26]$. The amplitudes of these azimuthal modulations depend on the kinematic variables relevant for the SIDIS process, namely the Bjorken scaling variable $x$, the 4-momentum of the virtual photon squared $Q^{2}$, the energy fraction of the hadron $z$, and the transverse momentum of the hadron $P_{T}$.

The first unambiguously measured single-spin phenomena in hard scattering, which triggered important theoretical developments, were transverse asymmetries measured in $p p$ collisions and siz- 
able longitudinal target $\left(A_{U L}^{\sin \phi}\right)$ and beam $\left(A_{L U}^{\sin \phi}\right)$ spin asymmetries observed at HERMES and JLab $[13,14,16,18,27,28]$ in SIDIS. Significant left-right asymmetries observed in the inclusive measurement of pions produced in the collision of transversely polarized (anti)protons with an unpolarized hydrogen target measured by the E-704 collaboration at center-of-mass energies of about $20 \mathrm{GeV}$, were confirmed at center-of-mass energies up to $200 \mathrm{GeV}$ by the STAR and BRAHMS collaboration at RHIC [21, 22].
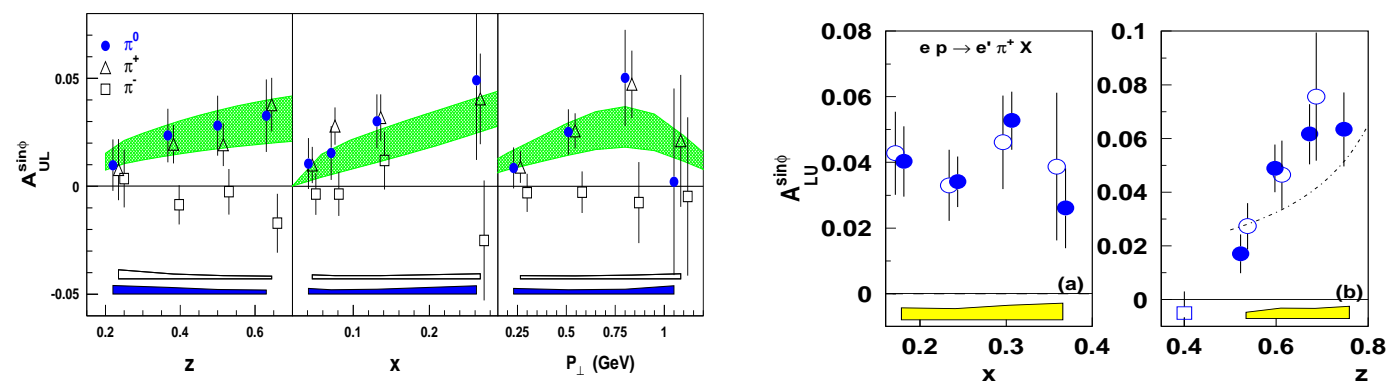

Figure 2. First measurements of SSAs in SIDIS with longitudinally polarized target [13] (left) and beam [18] (right).

Two fundamental QCD mechanisms giving rise to single-spin asymmetries were identified. First the Collins mechanism [29, 30], where the asymmetry is generated in the fragmentation of transversely polarized quarks, and second the Sivers mechanism [31-33], that arises due to final state interactions at the distribution function level. The first interpretation of observed significant SSA with longitudinally polarized target, $A_{U L}$, at HERMES[13] (see Fig. 2) was based on the convolution of the higher twist distribution function $h_{L}$, related to the production of transversely polarized quarks due to quark-gluon interactions and the leading-twist Collins fragmentation function [34, 35], $H_{1}^{\perp}$, describing the fragmentation of transversely polarized quarks to unpolarized hadrons (pions). The beam SSA, first measured by CLAS collaboration [18] (see Fig. 2, right panel) have also been interpreted in terms of the Collins mechanism [36-39] and used for a first determination of the twist-3 distribution function $e(x)$ [37]. The magnitude of the extracted $e(x)$ is also consistent with predictions using the chiral quark soliton model [40-42]. Collinear higher-twist distribution function $e(x)$ later on has been interpreted in terms of average transverse forces acting on a transversely polarized quark at the instant after absorbing the virtual photon [43].

The analysis [37, 44-46] of sub-leading single-spin asymmetries observed at HERMES [16] and later on at JLab [18] led to the introduction of a complete set of twist-3 distribution functions [33, 47]. Observation of the SSA in pion SIDIS has opened a new avenue to study the spin-orbit correlations and quark-gluon interactions, triggering studies of SSAs worldwide.

The first experimental evidence of a non-zero Collins function was obtained in SIDIS by the HERMES [15] and COMPASS collaborations [48] (see Fig. 3), where the convolution of the Collins function and the parton transversity distribution function $h_{1}$ was measured. Measurements performed at very different beam energies are consistent and indicate a significant asymmetry in the valence region with opposite signs for positive and negative pions. Recent measurements of multiplicities and double spin asymmetries as a function of the final transverse momentum of pions in SIDIS at JLab $[19,49]$ suggest that transverse momentum distributions may depend on the polarization of quarks and 
possibly also on their flavor. Kinematic dependencies of single- and double-spin asymmetries have been measured in a wide range in $x$ and $P_{T}$ with CLAS using a longitudinally polarized proton target. Measurements of the $P_{T}$-dependence of the double-spin asymmetry, performed for the first time, indicate the possibility of different average transverse momenta for quarks aligned or anti-aligned with the nucleon spin [49], consistent with latest lattice calculations of ratios of transverse momentum distributions of quarks anti-aligned $\left(q^{-}\right)$and aligned $\left(q^{+}\right)$with proton spin [50] (see Fig. 4).
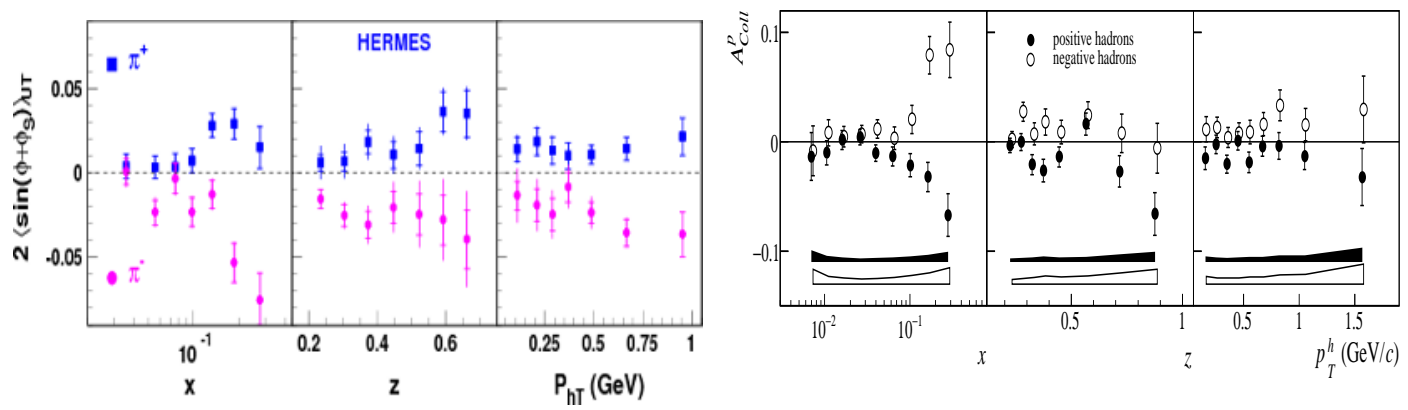

Figure 3. Dedicated measurements of Collins asymmetry with transversely polarized targets by the HERMES [15] (left plot) and COMPASS [48] (right plot) collaborations as a function of relevant kinematic variables.
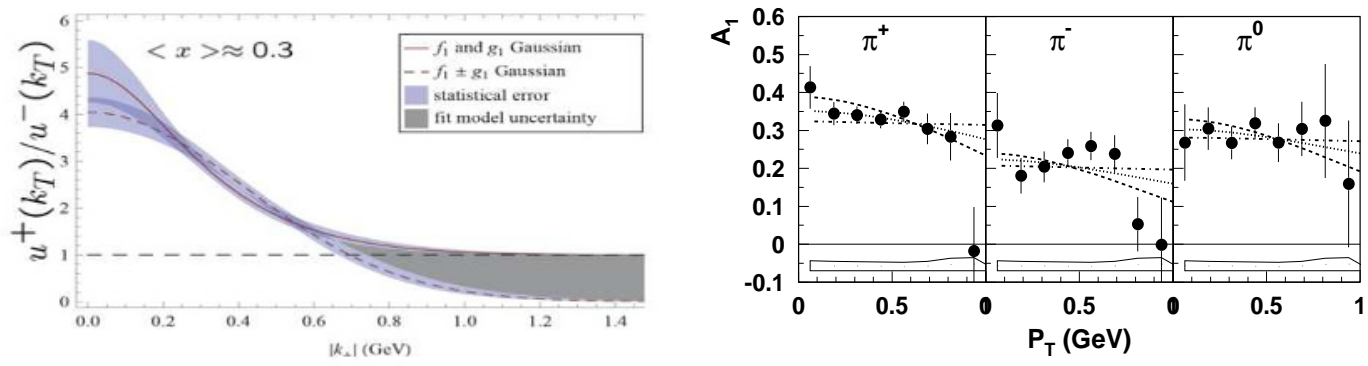

Figure 4. Transverse momentum dependence of ratio of densities of $u$ quarks anti-aligned $\left(q^{-}\right)$and aligned $\left(q^{+}\right)$ with the proton spin [50] (left panel). The solid curve and the statistical error band in blue have been obtained from the Gaussian, with gray band at the bottom showing the uncertainty due to the renormalization [50]. The dashed curve and the band were obtained using alternative Gaussian parametrizations. Two different ways to use Gaussians for the parametrization of lattice data, when positively defined $q^{+}=f_{1}+g_{1}$ and $q^{-}=f_{1}-g_{1}$ were parametrized, as opposed to $f_{1}$ and $g_{1}$ in the first case, measure possible model dependence. The right panel shows CLAS measurement of the $P_{T}$-dependence of the double spin asymmetry for pion SIDIS on a longitudinally polarized proton [49].

The direct information on the Collins function has been first obtained from $e^{+} e^{-}$annihilation experiments via the study of the semi-inclusive processes $e^{+} e^{-} \rightarrow q \bar{q} \rightarrow \pi \pi X$ at Belle [23] and later on confirmed by the BaBar experiment at SLAC [51]. Collins asymmetry can be extracted using the thrust reference frame (Fig. 5). Assuming the thrust axis defines the $q \bar{q}$ direction and by selecting pions in opposite hemispheres with respect to the thrust axis, the asymmetry as a function 
of azimuthal angles $\phi_{1}$ and $\phi_{2}$ of produced hadrons gives access to ratios of polarized and unpolarized fragmentation functions.
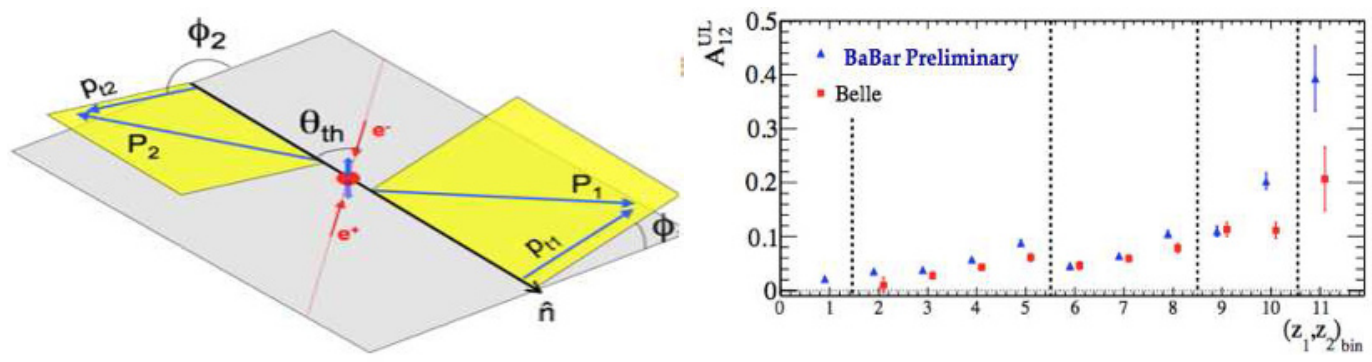

Figure 5. Kinematics of hadron production in $e^{+} e^{-}$collisions (left) and corresponding measurements of asymmetry related to the analyzing power of Collins fragmentation from BaBar and Bell experiments, and $z_{1}$ and $z_{2}$ are corresponding pion fractional energies.
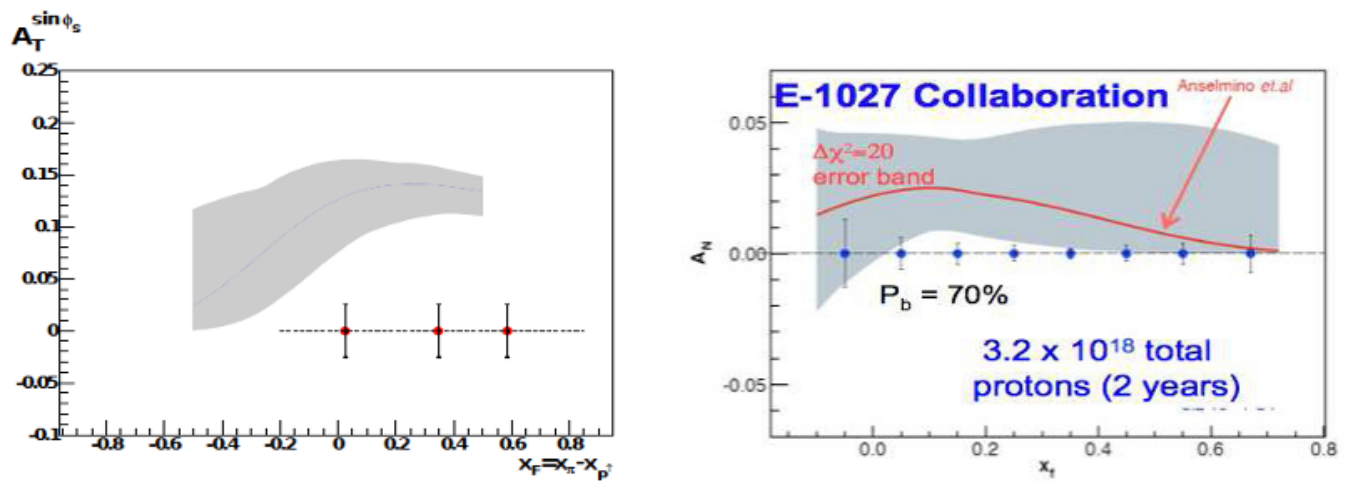

Figure 6. Expected statistical error of the Sivers asymmetry versus $x_{F}$ assuming two years of data taking (280 days) at COMPASS (left) and Fermilab (right) for a dimuon mass range of $4.2<M<8.5 \mathrm{GeV}$. The bands shows the theoretical predictions of the asymmetry from Anselmino et al. [52] with shaded areas showing the $\sqrt{20}$-sigma error band [53].

\section{Future measurements of DY and SIDIS}

One of the most remarkable features of the Sivers distribution function, responsible for the significant SSA with transversely polarized targets in SIDIS (see Fig. 3), where it appears in convolution with the unpolarized fragmentation function $D_{1}$, is the sign change from SIDIS to DY. An experimental proof of the sign-reversal property of the Sivers function is a crucial test of QCD in the non-perturbative regime.

Several experiments are currently planning to measure polarized Drell-Yan either with a polarized beam or a polarized target including COMPASS at CERN [54], Fermilab E1207 [53], Panda at FAIR [55], NICA at JINR [56], and RHIC. The main goal of COMPASS is to measure the interaction 
between valence quarks and valence antiquarks focusing on the pion-proton (deuteron) collisions, collecting a sufficient amount of data in the so-called safe dimuon invariant mass region from 4 to $9 \mathrm{GeV}$, where the theoretical formalism using TMDs is very well elaborated and the interpretation of the data is straightforward. The Fermilab E-906/SeaQuest spectrometer accommodates a large coverage in parton momentum fraction $x$, covering the valence quark region $(0.35-0.85)$, and the sea quark region $(0.1-0.45)$ for the beam and target polarizations, respectively (see Fig. 6).

Several proposals have been already approved by the JLab PAC to study GPDs and TMDs at JLab12 and were awarded the highest physics rating. The full program involves measurements using the CLAS12 and SOLID large acceptance detectors combined with precision measurements using Hall-C and Hall-A spectrometers. Precision measurements using the upgraded CLAS detector (CLAS12) with polarized $\mathrm{NH}_{3}$ and $\mathrm{ND}_{3}$ targets will allow access to the $k_{T}$-distributions of $u$ and $d$ quarks aligned and anti-aligned with the spin of the nucleon. Integrated over transverse momentum, the data will also be used to extract the $k_{T}$-integrated standard PDFs. A wider range in $Q^{2}$ provided by the CLAS12 detector at JLab would also allow studies of the $Q^{2}$-evolution, important for understanding and controlling possible higher-twist contributions. By using QCD evolved TMDs one can explain the observed discrepancies between HERMES [57] and COMPASS [58, 59] data, and predictions have been made for the non-trivial behavior of the Sivers asymmetry as a function of $Q^{2}$ [60]. Measuring the $Q^{2}$-dependence of the Sivers function is one of the main goals of the upgraded CLAS12 experiment using a transversely polarized HD target [1].

A combined analysis of JLab12 data sets from unpolarized, longitudinally polarized, and transversely polarized targets will allow to extract all relevant TMDs and GPDs that describe the 3D structure of the nucleon in the valence region.

\section{References}

[1] J. Dudek, R. Ent, R. Essig, K. Kumar, C. Meyer et al., Eur.Phys.J. A48, 187 (2012), 1208. 1244

[2] E. Aschenauer, A. Bazilevsky, K. Boyle, K. Eyser, R. Fatemi et al. (2013), 1304.0079

[3] A. Accardi, J. Albacete, M. Anselmino, N. Armesto, E. Aschenauer et al. (2012), 1212 . 1701

[4] X.D. Ji, Phys. Rev. Lett. 78, 610 (1997), hep-ph/9603249

[5] X. Ji, X. Xiong, F. Yuan, Phys.Rev.Lett. 109, 152005 (2012), 1202 . 2843

[6] C. Lorce, Phys.Rev. D87, 034031 (2013), 1205.6483

[7] M. Burkardt (2013), 1304.0281

[8] K. Goeke, M.V. Polyakov, M. Vanderhaeghen, Prog. Part. Nucl. Phys. 47, 401 (2001)

[9] M. Diehl, Phys. Rept. 388, 41 (2003), hep-ph/0307382

[10] S. Ahmad, G.R. Goldstein, S. Liuti, Phys. Rev. D79, 054014 (2009), 0805.3568

[11] S.V. Goloskokov, P. Kroll, Eur. Phys. J. C65, 137 (2010), 0906.0460

[12] S.V. Goloskokov, P. Kroll, Eur. Phys. J. A47, 112 (2011), 1106. 4897

[13] A. Airapetian et al. (HERMES), Phys. Rev. Lett. 84, 4047 (2000), hep-ex/9910062

[14] A. Airapetian et al. (HERMES), Phys. Rev. D64, 097101 (2001), hep-ex/0104005

[15] A. Airapetian et al. (HERMES), Phys. Rev. Lett. 94, 012002 (2005), hep-ex/0408013

[16] A. Airapetian et al. (HERMES), Phys. Lett. B648, 164 (2007), hep-ex/0612059

[17] V.Y. Alexakhin et al. (COMPASS), Phys. Rev. Lett. 94, 202002 (2005), hep-ex/0503002

[18] H. Avakian et al. (CLAS), Phys. Rev. D69, 112004 (2004), hep-ex/0301005

[19] H. Mkrtchyan et al., Phys. Lett. B665, 20 (2008), hep-ph/0709. 3020

[20] M. Osipenko et al. (CLAS), Phys. Rev. D80, 032004 (2009), hep-ex/0809. 1153

[21] J. Adams et al. (STAR), Phys. Rev. Lett. 92, 171801 (2004), hep-ex/0310058 
[22] I. Arsene et al. (BRAHMS), Phys. Rev. Lett. 101, 042001 (2008), nucl-ex/0801. 1078

[23] K. Abe et al. (Belle), Phys. Rev. Lett. 96, 232002 (2006), hep-ex/0507063

[24] J.J. Aubert et al. (European Muon), Phys. Lett. B130, 118 (1983)

[25] F. Giordano, R. Lamb (HERMES), AIP Conf. Proc. 1149, 423 (2009), hep-ex/0901 . 2438

[26] G. Sbrizzai (COMPASS) (2009), 0902.0578

[27] A. Airapetian et al. (HERMES), Phys. Lett. B562, 182 (2003), hep-ex/0212039

[28] A. Airapetian et al. (HERMES), Phys. Lett. B622, 14 (2005), hep-ex/0505042

[29] J.C. Collins, Nucl. Phys. B396, 161 (1993), hep-ph/9208213

[30] A. Kotzinian, Nucl. Phys. B441, 234 (1995), hep-ph/9412283

[31] D.W. Sivers, Phys. Rev. D41, 83 (1990)

[32] X. Ji, J. Ma, F. Yuan, Phys. Rev. D71, 034005 (2005), hep-ph/0404183

[33] J.C. Collins, A. Metz, Phys. Rev. Lett. 93, 252001 (2004), hep-ph/0408249

[34] K.A. Oganessian, H.R. Avakian, N. Bianchi, A.M. Kotzinian (1998), hep-ph/9808368

[35] A.M. Kotsinian, K.A. Oganesian, H. Avakian, E. De Sanctis, Nucl. Phys. A666, 290 (2000), hep-ph/9908466

[36] H. Avakian, A.V. Efremov, P. Schweitzer, F. Yuan (2008), hep-ph/0805 . 3355

[37] A.V. Efremov, P. Schweitzer, JHEP 08, 006 (2003), hep-ph/0212044

[38] A.V. Efremov, K. Goeke, P. Schweitzer, Eur. Phys. J. C24, 407 (2002), hep-ph/0112166

[39] L.P. Gamberg, G.R. Goldstein, K.A. Oganessyan, Phys. Rev. D67, 071504 (2003), hep-ph/0301018

[40] P. Schweitzer, Phys. Rev. D69, 034003 (2004), hep-ph/0307336

[41] M. Wakamatsu, Y. Ohnishi, Phys. Rev. D67, 114011 (2003), hep-ph/0303007

[42] Y. Ohnishi, M. Wakamatsu, Phys. Rev. D69, 114002 (2004), hep-ph/0312044

[43] M. Burkardt, hep-ph 0807.2599 (2008), hep-ph/0807. 2599

[44] A. Afanasev, C.E. Carlson (2003), hep-ph/0308163

[45] F. Yuan, Phys.Lett. B589, 28 (2004), hep-ph/0310279

[46] A. Metz, M. Schlegel, Eur. Phys. J. A22, 489 (2004), hep-ph/0403182

[47] D. Boer, P.J. Mulders, F. Pijlman, Nucl. Phys. B667, 201 (2003), hep-ph/0303034

[48] M.G. Alekseev et al. (The COMPASS), Phys. Lett. B692, 240 (2010), 1005.5609

[49] H. Avakian et al. (CLAS), Phys. Rev. Lett. 105, 262002 (2010), hep-ex/1003 . 4549

[50] B.U. Musch, P. Hagler, J.W. Negele, A. Schafer, Phys.Rev. D83, 094507 (2011), 1011.1213

[51] I. Garzia (BaBar Collaboration), Nuovo Cim. C034N06, 49 (2011)

[52] M. Anselmino, M. Boglione, U. D’Alesio, S. Melis, F. Murgia et al., Phys.Rev. D79, 054010 (2009), 0901.3078

[53] L. Isenhower, T. Hague, R. Towell, S. Watson, C. Aidala et al. (2012)

[54] O. Denisov (COMPASS Collaboration), Nuovo Cim. C035N2, 239 (2012)

[55] M. Lutz et al. (PANDA Collaboration) (2009), 0903. 3905

[56] A. Sissakian, O. Shevchenko, A. Nagaytsev, O. Ivanov, Eur.Phys.J. C59, 659 (2009), 0807.2480

[57] A. Airapetian et al. (HERMES), Phys. Rev. Lett. 103, 152002 (2009), 0906. 3918

[58] C. Adolph et al. (COMPASS Collaboration), Phys.Lett. B717, 383 (2012), 1205 . 5122

[59] C. Adolph et al. (COMPASS Collaboration), Phys.Lett. B717, 376 (2012), 1205.5121

[60] S.M. Aybat, A. Prokudin, T.C. Rogers, Phys.Rev.Lett. 108, 242003 (2012), 1112.4423 